\title{
Immune checkpoint inhibitors in patients with pretreated Hodgkin's lymphoma: a Korean single-center, retrospective study
}

\author{
Joon Young Hur ${ }^{1,2}$, Sang Eun Yoon ${ }^{1}$, Seok Jin Kim ${ }^{1}$, Won Seog Kim ${ }^{1}$ \\ ${ }^{1}$ Division of Hematology-Oncology, Department of Medicine, Samsung Medical Center, Sungkyunkwan University School of \\ Medicine, Seoul, ${ }^{2}$ Division of Hematology and Oncology, Department of Internal Medicine, Hanyang University Guri Hospital, Guri, \\ Korea
}

p-ISSN 2287-979X / e-ISSN 2288-0011 https://doi.org/10.5045/br.2020.2020014 Blood Res 2020;55:85-90.

Received on January 22, 2020

Revised on April 18, 2020

Accepted on June 11, 2020

\section{Correspondence to}

Won-Seog Kim, M.D., Ph.D. Division of Hematology-Oncology, Department of Medicine, Samsung Medical Center, Sungkyunkwan University School of Medicine, 81 Irwon-ro, Gangnam-gu, Seoul 06351, Korea

E-mail: wskimsmc@skku.edu

(C) 2020 Korean Society of Hematology

\section{Background}

Immune checkpoint inhibitors have demonstrated efficacy in the treatment of classical Hodgkin's lymphoma (cHL). We analyzed the efficacy and safety of pembrolizumab or nivolumab in patients with pretreated cHL.

Methods

Clinical data from the cancer chemotherapy registry of Samsung Medical Center were retrospectively analyzed to study patients with cHL treated with pembrolizumab or nivolumab between Oct 2015 and Dec 2018.

\section{Results}

Of the 20 patients, seven (35\%) were enrolled in the study after a relapse following autologous hematopoietic stem cell transplantation (ASCT) and $12(60 \%)$ after a relapse following receipt of brentuximab vedotin (BV). Sixteen (80\%) patients received pembrolizumab, and four (20\%) patients received nivolumab. The complete remission rate was $45 \%(9 / 20)$, and $30 \%(6 / 20)$ of patients achieved partial remission, for an overall response rate (RR) of 75\% [15/20; 95\% confidence interval (Cl), 34.7-93.3]. With a median follow-up duration of 14 months, the median PFS was 18 months (95\% Cl, 2.4-33.5 mo), and the median OS was 36 months [95\% Cl, 36-not applicable (NA) mo]. Pembrolizumab and nivolumab were generally well tolerated.

\section{Conclusion}

In this study, pembrolizumab and nivolumab both demonstrated clinical efficacy and tolerability in patients with cHL who failed previous chemotherapy or ASCT.

Key Words Pembrolizumab, Nivolumab, Hodgkin's lymphoma

\section{INTRODUCTION}

The incidence of classical Hodgkin's lymphoma (cHL) has been reported to be 0.4 per 100,000 person-years, constituting approximately $6 \%$ of all lymphomas in Korea [1]. Although the incidence of $\mathrm{cHL}$ was lower in Korea than in Western countries, the distributions of histological subtypes were similar [1]. To prevent T-cells from damaging normal cells, several immune system checkpoints exist, where undesirable immune responses can be inhibited or blocked [2]. One inhibitory immune checkpoint is the programmed cell death 1 (PD-1) pathway. Recently, PD-1 inhibitors including nivolumab and pembrolizumab were evaluated for treatment of patients with cHL showing disease progression during or following first-line chemotherapy [3]. The impressive results of nivolumab in relapsed and refractory cHL led to the U.S. Food and Drug Administration (FDA) approval in $2016[4,5]$. Prior to nivolumab, brentuximab vedotin (BV) was the only FDA approved cHL therapy after failure of autologous hematopoietic stem cell transplantation (ASCT), and there were no approved cHL therapies after failure of both ASCT and BV [6]. Another anti-PD-1 monoclonal antibody, pembrolizumab, was studied in a phase 1 trial published in 2016 (Keynote 013) [7]. A single-arm, phase II study of pembrolizumab in patients with relapsed or refractory $\mathrm{cHL}$ (KEYNOTE-087) showed that pembrolizumab was associated with high response rates and an acceptable safety profile 
in patients with relapsed or refractory cHL [8].

We analyzed the efficacy and safety of pembrolizumab or nivolumab in patients with cHL after previous chemotherapy or ASCT at a single cancer center institute. To our knowledge, this is the first real-world study to assess treatment patterns and outcomes in Korean patients with cHL.

\section{MATERIALS AND METHODS}

This study using the cancer registry of Samsung Medical Center (SMC) was retrospectively reviewed and approved by the SMC Institutional Review Board (Seoul, Korea). All patients gave written informed consent prior to starting therapy, according to institutional guidelines. We reviewed the medical records of patients with $\mathrm{cHL}$ who were treated with pembrolizumab or nivolumab as second-line or later therapy between Oct 2015 and Dec 2018. The eastern cooperative oncology group (ECOG) performance status was also assessed. Criteria for study inclusion were as follows: 1) histologically confirmed diagnosis of cHL arising from lymph node and/or extranodal organs, 2) 18 years of age or older, and 3) ASCT or prior chemotherapy such as ABVD (doxorubicin, bleomycin, vinblastine, dacarbazine) and $\mathrm{BV}$.

Pembrolizumab 100 or $200 \mathrm{mg}$ was administered intravenously every three weeks, and nivolumab $3 \mathrm{mg} / \mathrm{kg}$ was administered intravenously every two weeks. During the treatment period, laboratory and imaging assessments were performed. The laboratory assessments included complete blood count $(\mathrm{CBC})$ with differential, serum urea, serum creatinine, aspartate aminotransferase (AST), alanine aminotransferase (ALT), total bilirubin, alkaline phosphatase, lactate dehydrogenase (LDH), and Epstein-Barr virus (EBV). The immunohistochemistry (IHC) staining of programmed cell death - ligand 1 (PD-L1) 22C3 using monoclonal mouse anti-PD-L1 clone 22C3 was reviewed. The PD-L1 tumor proportion score (TPS) was calculated as the percentage of viable tumor cells showing partial or complete membrane staining at any intensity. Positron emission tomography (PET) and computed tomography (CT) scans of all involved sites were evaluated before starting PD-1 inhibitors. To evaluate clinical response to nivolumab or pembrolizumab, patients underwent CT or PET scans at weeks 12, 24, and 36 , or 60 weeks. We evaluated efficacy assessments using the revised response criteria for malignant lymphomas [9]. The best overall response was defined as the best response between the date of the first dose and the last efficacy assessment before subsequent therapy. Pembrolizumab or nivolumab was continued until complete response, disease progression, or excessive toxic effects.

The primary endpoint of the present study was overall response rate $(\mathrm{RR})$. Secondary endpoints were progression-free survival (PFS), overall survival (OS), and safety. The time from the first day of pembrolizumab or nivolumab administration to the date of documented disease progression or death was considered PFS. The time from the first day of pembrolizumab or nivolumab administration to the date of death was considered OS. Cox proportional hazards model was used in univariate analysis to identify significant prognostic factors for PFS and OS. All $P$-values were two-sided, with $P<0.05$ indicating statistical significance. All analyses were performed using $\mathrm{R}$ for Windows v3.3.2 software ( $\mathrm{R}$ Core Team, Vienna, Austria; http://www.r-project.org).

\section{RESULTS}

Patient characteristics are given in Table 1. As shown, among the 20 patients, $65 \%$ were men, and the median age was 34 years (range, 22-79). There were 4 patients aged 65 or older. Medical records from 16 eligible patients who were treated with pembrolizumab and 4 patients with nivolumab for relapsed or refractory cHL were reviewed. Of those

Table 1. Patient characteristics.

\begin{tabular}{|c|c|c|}
\hline & $\mathrm{N}$ & $\%$ \\
\hline \multicolumn{3}{|l|}{ Age, years } \\
\hline Median (range) & $34(22-79)$ & \\
\hline Age $\geq 65$ & 4 & 20 \\
\hline \multicolumn{3}{|l|}{ Sex } \\
\hline Male & 13 & 65 \\
\hline Female & 7 & 35 \\
\hline \multicolumn{3}{|l|}{ Histology } \\
\hline Nodular sclerosis & 9 & 45 \\
\hline Mixed cellularity & 6 & 30 \\
\hline Subtype indeterminate & 5 & 25 \\
\hline \multicolumn{3}{|l|}{ ECOG performance status } \\
\hline 0 & 12 & 60 \\
\hline 1 & 5 & 25 \\
\hline 2 & 3 & 15 \\
\hline \multicolumn{3}{|l|}{ EBV in-situ hybridization } \\
\hline Negative & 6 & 30 \\
\hline Positive & 7 & 35 \\
\hline Unknown & 7 & 35 \\
\hline \multicolumn{3}{|l|}{ B symptom } \\
\hline No & 18 & 90 \\
\hline Yes & 2 & 10 \\
\hline \multicolumn{3}{|l|}{ Stage } \\
\hline I & 1 & 5 \\
\hline II & 4 & 20 \\
\hline III & 6 & 30 \\
\hline IV & 9 & 45 \\
\hline \multicolumn{3}{|l|}{ Extranodal involvement } \\
\hline Lung & 9 & 45 \\
\hline Bone & 7 & 35 \\
\hline Liver & 2 & 10 \\
\hline Prior ASCT & 7 & 35 \\
\hline Prior radiotherapy, No. & 4 & 20 \\
\hline Prior brentuximab vedotin, No. & 12 & 60 \\
\hline \multicolumn{3}{|l|}{ Prior chemotherapies, No. } \\
\hline $1-3$ & 14 & 70 \\
\hline$\geq 4$ & 6 & 30 \\
\hline
\end{tabular}

Abbreviations: ASCT, autologous hematopoietic stem cell transplantation; EBV, Epstein-Barr virus; ECOG, eastern cooperative oncology group. 
treated with pembrolizumab, 10 had been treated with pembrolizumab $100 \mathrm{mg}$ every 3 weeks and 6 with pembrolizumab $200 \mathrm{mg}$. Efficacy did not differ significantly between the doses. Of 20 patients, most had been treated with ABVD chemotherapy (90\%), 12 (60\%) received BV before immunotherapy, and 7 (35\%) had relapsed/refractory disease after ASCT. Only 3 patients had available data on PD-L1 expression: one patient with PD-L1 (22C3) 100\%, and the others with PD-L1 (22C3) 50\% and 40\%, respectively. Extranodal disease involving lung, bone, liver, or pleura was found in $70 \%$ of the patients $(14 / 20)$.

Patients received pembrolizumab for a total of 171 cycles (median, 13; range, 1-24) and nivolumab for 67 cycles (median, 15; range, 5-24). Of 20 patients, nine showed complete responses to immunotherapy (45\%) and six showed partial responses (30\%). The objective response rate was 75\% [15/20; 95\% confidence interval (CI), 34.7-93.3]. The maximum reduction in tumor burden from baseline for each patient is shown in Fig. 1A. Of 9 patients with complete response, 7 had received previous treatment with brentuximab. Of the 15 patients who had a complete or partial response, $4(26.6 \%)$ had the first response by 10 weeks (range, 8 to $16 \mathrm{wk}$ ) (Fig. 1B). At the time of data collection with a median follow-up duration of 14 months, the median PFS was 18 months (95\% CI, 2.4-33.5, Fig. 2A), and the median OS was 36 months (95\% CI, 36-NA mo, Fig. 2B).

Of the 11 patients $(55 \%)$ who discontinued treatment, $2(10 \%)$ had complete remission, 1 (5\%) had toxic effects (cardiac myocarditis), 7 (35\%) had progressive disease during treatment, and 1 (5\%) elected to undergo ASCT. Two patients showed complete remission after pseudoprogression when treated with pembrolizumab. Univariate analysis results for PFS and OS are shown in Table 2. PFS was not significantly influenced by age, performance status, EBV, prior ASCT, or type of PD-1 inhibitors (pembrolizumab vs. nivolumab). Univariate analysis revealed that ECOG performance status (0-1 vs. 2) was a significant prognostic factor for overall survival $(P=0.016)$.

Pembrolizumab or nivolumab was generally well tolerated. The most common treatment-related adverse events (AEs) (Table 3) were fever (15\%), aminotransferase increase (10\%), fatigue $(5 \%)$, and neutropenia (5\%). Immune-related AEs resulting in treatment discontinuation were observed in 2 patients who both received steroids. Of 20 patients, one had pembrolizumab-associated myocarditis. After pembrolizumab was administered for 10 cycles, a 32-year-old woman presented with new, progressive dyspnea that had developed during the previous week. Echocardiography revealed pericardial and pleural effusion. Heart biopsy revealed lymphohistiocytic infiltration with myocyte damage, con-

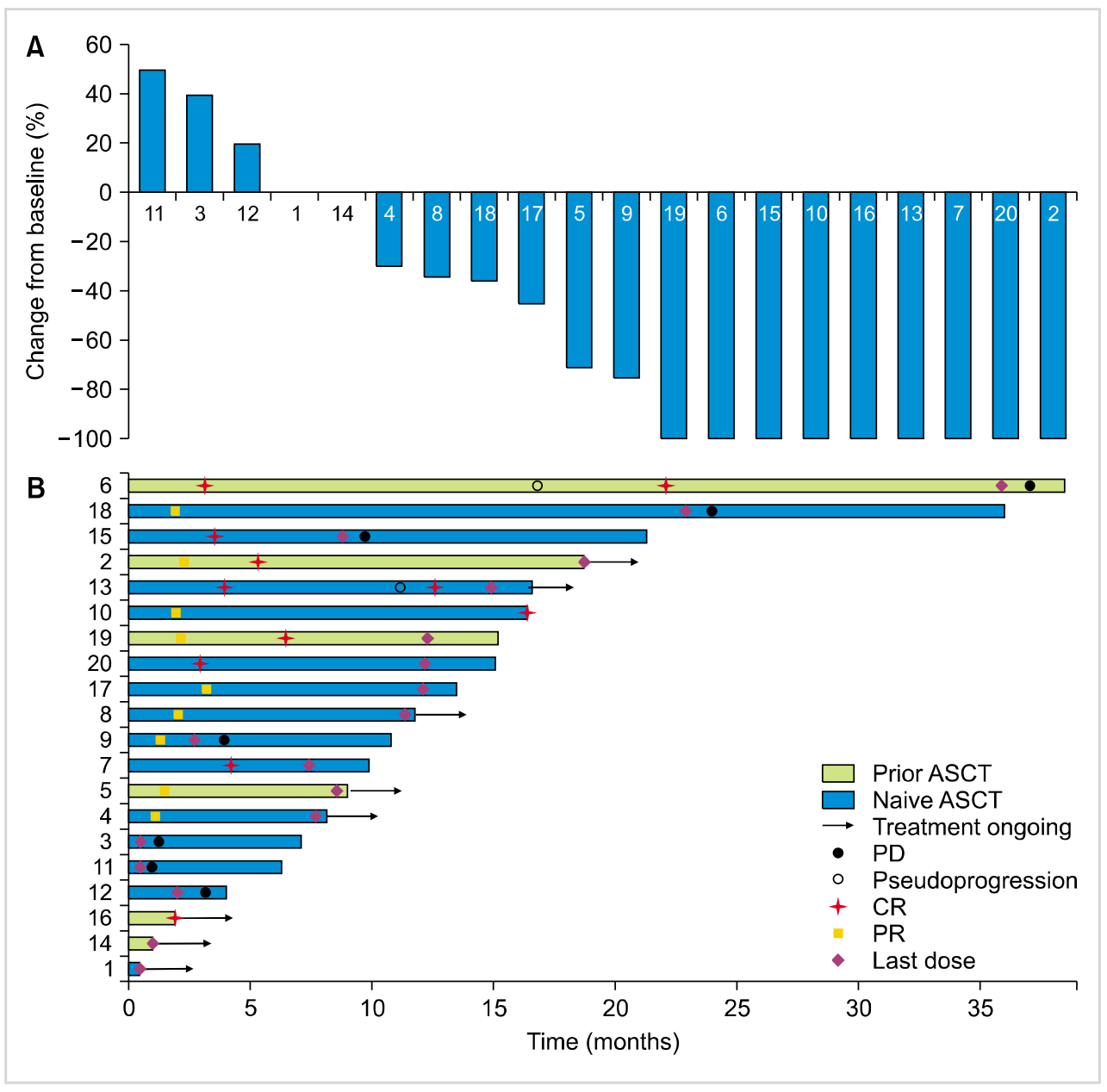

Fig. 1. Responses shown as (A) waterfall plot and (B) swimmer plot. 

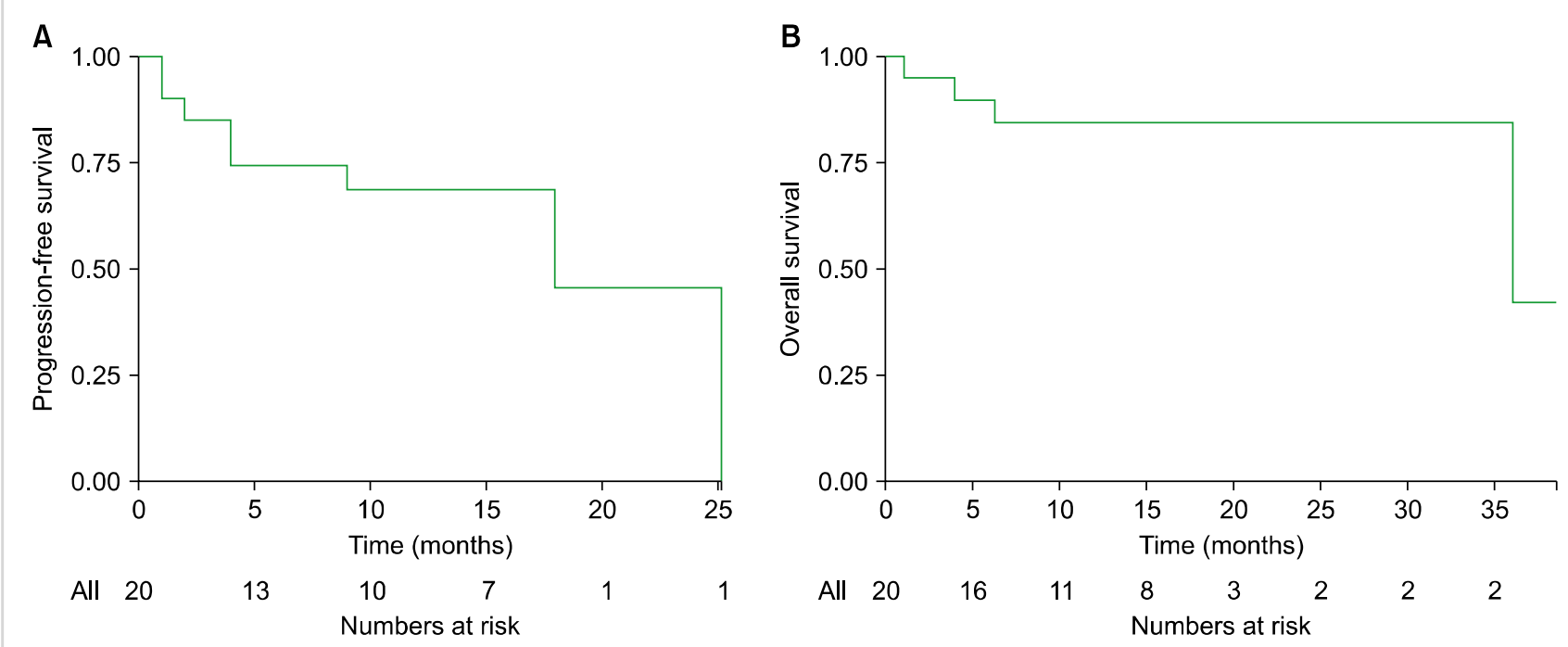

Fig. 2. Kaplan-Meier curves of progression-free survival (A) and overall survival (B) for all patients.

Table 2. Univariate analysis of prognostic factors for survival.

\begin{tabular}{|c|c|c|c|c|c|c|}
\hline \multirow{2}{*}{ Variables } & \multicolumn{3}{|c|}{ PFS } & \multicolumn{3}{|c|}{ OS } \\
\hline & $\mathrm{HR}$ & $95 \% \mathrm{Cl}$ & $P$ & $H R$ & $95 \% \mathrm{Cl}$ & $P$ \\
\hline Age, $\geq 65$ vs. $<65$ & 0.52 & $0.06-4.38$ & 0.549 & 2.74 & $0.36-21.03$ & 0.332 \\
\hline EBV, positive vs. negative & 0.28 & $0.03-2.72$ & 0.270 & 0.54 & $0.05-5.95$ & 0.613 \\
\hline ECOG, 0 or 1 vs. 2 & 2.31 & $0.43-12.54$ & 0.331 & 18.81 & $1.72-206.00$ & 0.016 \\
\hline Prior ASCT, yes vs. no & 0.56 & $0.10-3.03$ & 0.505 & 0.56 & $0.06-5.55$ & 0.622 \\
\hline Pembrolizumab vs. nivolumab & 0.58 & $0.11-3.18$ & 0.530 & 0.53 & $0.05-5.19$ & 0.609 \\
\hline
\end{tabular}

Abbreviations: AASCT, autologous hematopoietic stem cell transplantation; $\mathrm{Cl}$, confidence interval; EBV, Epstein-Barr virus; ECOG, eastern cooperative oncology group; $\mathrm{HR}$, hazard ratio; OS, overall survival; PFS, progression free survival.

Table. 3. Most commonly observed adverse events $(\mathrm{N}=20)$.

\begin{tabular}{lccccc}
\hline & \multicolumn{2}{c}{ All grades } & & \multicolumn{2}{c}{ Grade 3 or 4} \\
\cline { 2 - 3 } \cline { 5 - 6 } & No. & $\%$ & & No. & $\%$ \\
\hline Fever & 3 & 15 & & 0 & 0 \\
Aminotransferase increase & 2 & 10 & & 0 & 0 \\
Fatigue & 1 & 5 & 0 & 0 \\
Neutropenia & 1 & 5 & & 5 \\
Thrombocytopenia & 1 & 5 & 0 & 0 \\
Pneumonitis & 1 & 5 & 1 & 5 \\
Myocarditis & 1 & 5 & 1 & 5 \\
Hypothyroidism & 1 & 5 & 0 & 0 \\
Nausea & 1 & 5 & 0 & 0 \\
Vomiting & 1 & 5 & 0 & 0 \\
\hline
\end{tabular}

sistent with lymphocytic myocarditis. She recovered rapidly after steroid therapy. There were no grade 4 treatment-related AEs and no deaths related to immunotherapy.

\section{DISCUSSION}

This study analyzed the clinical outcomes of PD-1 immunotherapy in patients with cHL who had been treated with previous chemotherapy or ASCT. Although conventional therapy (i.e., ABVD, BV) for cHL is associated with a high rate of cure (80\%), up to $20 \%$ of patients will have relapsed or refractory disease [10]. In heavily pretreated patients with relapsed or refractory $\mathrm{cHL}$, the majority of whom experienced relapse after ASCT and BV treatment, the use of nivolumab was associated with an overall response rate of $66-87 \%$ and a rate of PFS of $77-86 \%$ at 24 weeks [5, 11]. We await the results of randomized studies such as the ongoing phase III study comparing brentuximab with nivolumab in combination versus brentuximab alone in patients with relapsed/refractory cHL (NCT03138499). Several ongoing trials are investigating the efficacy of pembrolizumab in various settings, including a phase III head-to-head trial of pembrolizumab and brentuximab in patients with relapsed/refractory cHL (NCT 02684292).

As far as we know, this is the first real world study 
of PD-1 inhibitors in Korean patients with cHL. In 2015 , when immunotherapy was introduced into clinical practice of cHL, some patients with cHL in this study were treated with low-dose pembrolizumab. A previous study showed that low-dose pembrolizumab (median dose $100 \mathrm{mg}$; range, 100-200 mg, every $3 \mathrm{wk}$ ) was highly efficacious, achieving responses with minimal toxicity and at lower costs [12].

Some patients who has an increase in the size of lesions at response assessment were noted to have late response [13]. The previous studies described these unique response patterns, termed pseudoprogression [14]. An analysis of pembrolizumab in patients with advanced melanoma found that 3.6\% (seven of 192 patients) experienced progressive disease at first assessment, followed by clinical response at second assessment [15]. In this study, two patients had definite clinical improvement despite imaging data indicating progression. Clinicians should be aware of the potential for pseudoprogression in patients showing clinical improvement.

Seven $(35 \%)$ patients had progressive disease during treatment. One of them was transformed into diffuse large B cell lymphoma (DLBCL) and treated with RCHOP (rituximab, cyclophosphamide, doxorubicin, vincristine, and prednisone). Of seven patients, two received ASCT after PD-1 inhibitors, but both showed progression. Three patients were treated with another PD-1 inhibitor, and one patient died of aspiration pneumonia.

The exact mechanism of PD-1 inhibitors in cHL remains unknown, and more research is needed. In cHL, alterations in chromosome 9p24.1 increase the abundance of PD-1 ligands and promote their induction through the Janus kinasesignal transducer and activator of transcription signaling, defining the PD-1 pathway as a rational therapeutic target [16].

Immune checkpoint blockade can have adverse inflammatory effects, which are often termed immune-related adverse events, involving the gastrointestinal tract, endocrine glands, skin, liver, lung, and heart [17]. Immune-mediated pneumonitis, colitis, hepatitis, hypophysitis, and thyroiditis are less common toxic effects of PD-1 antibody [18]. Surprisingly, there was a life-threatening or fatal treatment-related event (myocarditis) in this study. She is now regularly watching the progress without further chemotherapy with a complete remission. There are rare cases of immune checkpoint inhibitor-induced myocarditis [19]. As a result of recent data, in heavily pretreated disease, immune checkpoint inhibitors are moving to earlier lines of treatment [20].

In this study, pembrolizumab or nivolumab demonstrated clinical efficacy and tolerability in patients with cHL who failed previous chemotherapy or ASCT. Further investigations of immune checkpoint inhibitors and combinatory approaches in relapsed or refractory $\mathrm{cHL}$ are needed.

\section{ACKNOWLEDGMENTS}

This study was conducted ethically in accordance with the World Medical Association Declaration of Helsinki. Seok
Jin Kim and Won Seog Kim contributed to the study conception and design. Joon Young Hur and Sang Eun Yoon contributed to revision of the manuscript. Joon Young Hur contributed to acquisition of data and drafting of manuscript. All authors read and approved the final manuscript.

\section{Authors' Disclosures of Potential Conflicts of Interest}

No potential conflicts of interest relevant to this article were reported.

\section{REFERENCES}

1. Won YW, Kwon JH, Lee SI, et al. Clinical features and outcomes of Hodgkin's lymphoma in Korea: Consortium for Improving Survival of Lymphoma (CISL). Ann Hematol 2012;91:223-33.

2. De Re V, Caggiari L, Repetto O, Mussolin L, Mascarin M. Classical Hodgkin's lymphoma in the era of immune checkpoint inhibition. J Clin Med 2019;8:1596.

3. Bröckelmann PJ, Engert A. Checkpoint inhibition in Hodgkin lymphoma - a review. Oncol Res Treat 2017;40:654-60.

4. Kasamon YL, de Claro RA, Wang Y, Shen YL, Farrell AT, Pazdur R. FDA approval summary: nivolumab for the treatment of relapsed or progressive classical Hodgkin lymphoma. Oncologist 2017;22:585-91.

5. Ansell SM, Lesokhin AM, Borrello I, et al. PD-1 blockade with nivolumab in relapsed or refractory Hodgkin's lymphoma. N Engl J Med 2014;372:311-9.

6. de Claro RA, McGinn K, Kwitkowski V, et al. U.S. Food and Drug Administration approval summary: brentuximab vedotin for the treatment of relapsed Hodgkin lymphoma or relapsed systemic anaplastic large-cell lymphoma. Clin Cancer Res 2012;18:5845-9.

7. Armand P, Shipp MA, Ribrag V, et al. Programmed death-1 blockade with pembrolizumab in patients with classical Hodgkin lymphoma After brentuximab vedotin failure. J Clin Oncol 2016; 34:3733-9.

8. Chen R, Zinzani PL, Fanale MA, et al. Phase II study of the efficacy and safety of pembrolizumab for relapsed/refractory classic Hodgkin lymphoma. J Clin Oncol 2017;35:2125-32.

9. Cheson BD, Pfistner B, Juweid ME, et al. Revised response criteria for malignant lymphoma. J Clin Oncol 2007;25:579-86.

10. Bair SM, Mato A, Svoboda J. Immunotherapy for the treatment of Hodgkin lymphoma: an evolving paradigm. Clin Lymphoma Myeloma Leuk 2018;18:380-91.

11. Younes A, Santoro A, Shipp M, et al. Nivolumab for classical Hodgkin's lymphoma after failure of both autologous stem-cell transplantation and brentuximab vedotin: a multicentre, multicohort, single-arm phase 2 trial. Lancet Oncol 2016;17:1283-94.

12. Chan TSY, Luk TH, Lau JSM, Khong PL, Kwong YL. Low-dose pembrolizumab for relapsed/refractory Hodgkin lymphoma: high efficacy with minimal toxicity. Ann Hematol 2017;96: 647-51.

13. Seymour L, Bogaerts J, Perrone A, et al. iRECIST: guidelines for response criteria for use in trials testing immunotherapeutics. Lancet Oncol 2017;18:e143-52. 
14. Hong J, Bae J, Lee SG, et al. Complete Remission after pseudoprogression in refractory classical Hodgkin lymphoma treated with pembrolizumab. Korean J Med 2017;92:415-8.

15. Chiou VL, Burotto M. Pseudoprogression and immune-related response in solid tumors. J Clin Oncol 2015;33:3541-3.

16. Green MR, Monti S, Rodig SJ, et al. Integrative analysis reveals selective 9p24.1 amplification, increased PD-1 ligand expression, and further induction via JAK2 in nodular sclerosing Hodgkin lymphoma and primary mediastinal large B-cell lymphoma. Blood 2010;116:3268-77.

17. Postow MA, Sidlow R, Hellmann MD. Immune-related adverse events associated with immune checkpoint blockade. N Engl J Med 2018;378:158-68.

18. Topalian SL, Hodi FS, Brahmer JR, et al. Safety, activity, and immune correlates of anti-PD- 1 antibody in cancer. N Engl J Med 2012;366:2443-54.

19. Mahmood SS, Fradley MG, Cohen JV, et al. Myocarditis in patients treated with immune checkpoint inhibitors. J Am Coll Cardiol 2018;71:1755-64.

20. Vassilakopoulos TP, Chatzidimitriou C, Asimakopoulos JV, et al. Immunotherapy in Hodgkin lymphoma: present status and future strategies. Cancers (Basel) 2019;11:1071. 